Part 1. General Sky Survey Reviews 


\title{
THE PROSPECTS OF LARGE SURVEYS IN ASTRONOMY
}

\author{
M. HARWIT \\ 511 H Street, SW, Washington, DC, 20024-2725
}

\begin{abstract}
Major astronomical discoveries of the past have been closely linked with the implementation of strikingly powerful new observational techniques in astronomy. Surveys, in general, were not as likely to lead to the discovery of new phenomena. In recent years, however, many farreaching instrumental advances have been incorporated into observatories in space, whose mission, in part, was to conduct unbiased surveys. Here, we provide a review of some of the successes of earlier surveys, with the purpose of identifying the relative extent to which increased instrumental capabilities, as contrasted to increased sky coverage or numbers of sources observed, may be expected to lead to the discovery of new phenomena. A similar comparison can be made of the extent to which these two approaches-emphasis on instrumental capabilities, as contrasted to emphasis on sky coveragealso contribute to an increase in astrophysical understanding. Here, the distinction needs to be made between discovering a new phenomenon and understanding its underlying processes.
\end{abstract}

\section{The Nature of Astronomical Discovery}

The almost ubiquitous role of powerful new technologies in revealing new cosmic phenomena is by now well established (Harwit 1981). Access to entirely new wavelength domains in the electromagnetic spectrum, led to the discovery of a microwave background dating back to the first million years in the evolution of the cosmos; galaxies far more luminous at infrared wavelengths than in all other spectral ranges combined; massive X-ray haloes surrounding the central galaxies of large clusters; and bursts of gamma rays, possibly emanating from sources more luminous than any others known in the universe. 
Access to extremely high spatial resolving powers in radio astronomy led first to the discovery of quasi-stellar radio sources (quasars) and, as radio-interferometric resolving powers increased even further, to the discovery of superluminal sources. Still in the radio regime, great strides in spectral resolving power gave us stellar and interstellar masers, while improvements in time resolution, by many orders of magnitude, showed up the existence of pulsars. Similar discoveries resulted from thousand-fold improvements in spatial, spectral and temporal resolving powers in other wavelength domains.

Such discoveries often were made virtually as soon as the novel capabilities essential to making the requisite observations were introduced into astronomy. Seldom were these initial exciting findings followed by the discovery of other comparably significant phenomena. The rule of thumb appeared to be, that new phenomena were discovered quickly following the introduction into astronomy of techniques that went beyond the state of the art by three or more orders of magnitude-either in access to new spectral wavelength regimes or, within a given regime, to increased spatial, spectral or temporal resolving power.

It is not as though a new technique becomes useless after its initial flash of success. Rather, it joins an increasingly comprehensive kit of astronomical tools that help us to probe and analyze the emission from astronomical sources to improve our understanding of them.

There is reason to believe that large surveys, capable of increasing the number of sources studied by many orders of magnitude, may similarly uncover new phenomena. In particular, rare phenomena or faint sources overshadowed by millions of others that are more prevalent or luminous might be discovered only in this fashion. If the number of sources studied can be increased a thousand or a million-fold, major new phenomena may be expected to show up. The comprehensive surveys now being planned often are designed to measure hundreds of millions of sources-many orders of magnitude more, in most spectral ranges, than available before. The chances of discovering rare phenomena thus come into range.

\section{Monochromatic, Polychromatic, or Multispectral Surveys}

Monochomatic surveys, such as the early optical surveys of the nineteenth century provided useful sky charts and catalogues that listed accurate positions and approximate magnitudes of sources. Such surveys limited the insights one could gain on differences characterizing the different sources. Point sources could be identified only by their different magnitudes, unless they were repeatedly observed, which was time consuming until photographic plates were introduced in the latter part of the century. In 1899, 
S. I. Bailey of Harvard University made photographic observations of the globular clusters Messier 5 from a site in Arequipa, Peru. One might not think of this as a survey, but he was simultaneously observing somewhere between a hundred thousand and a million stars. In comparing different plates, he was surprised to see that the magnitude of quite a number of stars had changed. He had discovered the existence of short-period cluster variables.

In the 1960 s, the two-micron infrared sky survey conducted by Neugebauer and Leighton (1969) was seminal in identifying whole new varieties of sources of radiation that emitted powerfully in the near infrared. But the survey's full implications could be understood only by identifying individual sources, largely with the aid of far deeper optical surveys, particularly with the comprehensive Palomar Sky Survey plates that covered the same portions of the sky.

Roughly forty years apart, the Palomar Sky Survey and the Hipparcos mission have provided important two-color capabilities in the optical range. The two-color discrimination was helpful for many purposes. But positional identification was perhaps the most useful feature of the Palomar plates, as far as radio, infrared, or X-ray astronomers were concerned. They helped to identify a source as a star, a planetary nebula, or a galaxy, and often could provide more detailed descriptions of the nature of the object within its class. In contrast, the Hipparcos mission's greatest benefit for most astronomers is the unprecedented positional accuracy it has provided. Its positional and parallax accuracy are in the range of 0.0013 and 0.0015 arc seconds. Its proper motion measures on roughly 100,000 stars are accurate to 0.0018 arc seconds per year.

Did the Palomar plates or the Hipparcos missions lead to the discovery of new phenomena? By themselves, probably not, though they are massive surveys. But their contributions to understanding are undeniable. The Hipparcos mission has already shown that an accurate measure of distance provides a far better defined Herzsprung-Russell diagram for stars local to the solar neighborhood. Stellar properties apparently vary significantly less among these stars than might have been previously thought. Once a star's $\mathrm{B}-\mathrm{V}$ coloration is known, its absolute magnitude on the main sequence or on the red giant branch, is quite narrowly defined (ESA 1996).

The two-micron survey's successors, the DENIS survey just starting in the south, and the all-sky 2-MASS survey about to begin in the north, will each conduct observations at three near-infrared wavelengths, and will detect in excess of a hundred million stars and 500,000 galaxies. All sightings will be cross-correlated with other wavelength ranges. The spectrophotometric classifications to be obtained in this way, may provide the characterizing features that will permit us to discover new phenomena. 
Polychromatic surveys have greater potential for differentiation and discovery of new kinds of sources than their monochromatic counterparts. The IRAS four-color survey provided us with clear differentiation between infrared sources bright predominantly at the shorter $12 \mu \mathrm{m}$ or at the longer $100 \mu \mathrm{m}$ end of the spectral range. Even here, however, additional insights and true discoveries came about only through comparison to observations obtained in entirely different spectral ranges. Thus, we became aware of the existence of ultraluminous, $L \geq 10^{13} L_{\odot}$ extragalactic sources, mostly identified with colliding galaxies undergoing a burst of star formation, and dust clouds around stars, possibly remnants of proto-planetary dust clouds. In both these instances, the pre-existence of much more comprehensive optical surveys was essential to the recognition that something new might be at play.

\section{Specialized Capabilities for Revealing Unprobed Characteris- tics}

It is becoming clear that, at the current state of development in astronomy, where virtually every part of the electromagnetic spectrum has been at least superficially probed, it is no longer possible to make discoveries in a single spectral band alone. Such early discoveries as quasi-stellar radio sources, far more compact than the largely extended sources previously known, or radio sources that pulsated at incredibly regular rates, or a ubiquitous $\mathrm{X}$ ray background that was clearly different from point-like X-ray sources, or a bright microwave background - all of which were remarkable in their own rights without even identifying corresponding optical features-may soon become matters of the past. Within a given spectra range, the only remaining, broad-band investigations that might uncover new phenomena would be extremely high energy gamma ray observations or a search for extremely fast pulsations. Somewhat surprisingly, the optical band is one domain in which opportunities for fast timing have never existed. Atmospheric interference makes short term observations from the ground unreliable, and no spacecraft mission has incorporated that capability. A survey with such instrumental capabilities could well uncover previously unknown phenomena.

\section{Uneven Coverage}

The difficulty at the moment is the very uneven sensitivity and angular and spectral resolution capabilities in the different spectral ranges. The Compton Gamma Ray Observatory (CGRO) has permitted us to make great strides forward. But, in terms of the number of sources observed, it has only brought us to the point to which the Uhuru X-ray survey had brought 
X-ray astronomy two decades ago. The IRAS survey gave us access to hundreds of thousands of sources, but its spatial resolution was only at the arc minute level, a far cry from optical or radio spatial resolving powers. In the far-infrared and X-ray domains advances in spectral resolving power are providing increasing capabilities in identifying grains and molecular, atomic and ionic species across a wide set of temperature and pressure ranges. Our recognition and understanding of the physical and chemical processes at work are thus vastly improved. By itself, high resolution spectroscopy can also be an engine of discovery. The early detection of interstellar and circumstellar masers through high-resolution radio spectroscopy is one case in point. But the analytic virtues of spectroscopy ultimately may prove far more significant than the ability to discover startling new phenomena.

Each generation builds more powerful instruments, but so far the mismatch between capabilities in the different spectral regions is enormous. For CGRO scientists the obvious frustration is the inability to locate the tantalizing gamma-ray bursts with the arc second or sub-arc second spatial precision that would be needed to identify potential sources on optical or radio maps.

\section{Discovering Rare $\mathbf{P h e n o m e n a}$}

The discovery of phenomena that are exceedingly rare, occurring perhaps even less frequently than supernovae or gamma-ray bursts, may require new kinds of surveys. To date, we have not undertaken long-duration surveys, where evolutionary effects or seldom-occurring, faint, nearby phenomena could be discovered. Most of our surveys have stopped after only a few years. Sometimes their endurance is linked to the life-work of an individual; in other instances, novel techniques overtake observations already in hand, making them outdated.

A trade-off exists between long-duration surveys that await rare occurrences, and greater sensitivity investigations, which enable us to detect these same occurrences at greater distances and, thereby, at more frequent intervals. This trade-off may, however, have limited applicability. If gamma-ray bursts turn out to be cosmological, as now seems likely, and if they actually have a threshold energy below which they are not generated, then increasing sensitivity will not be of help in registering larger numbers of bursts. If there exist phenomena that occur thousands or millions of times less frequently than gamma-ray bursts but still play powerful roles in cosmic evolution, then we may need to mount surveys that persist many decades or centuries to discover them. We have not yet learned how to do that. 
Gamma-ray astronomers have been fortunate in having a relatively empty sky against which to observe their bursts. Imagine how much more difficult it would be to mount a survey to search for rare pulsations in a sky filled with hundreds of millions of sources all variable, at some level, intrinsically or because of interstellar scintillation.

\section{Data Rates}

Consider the search for a faint set of sources that might radiate appreciably in only two broad spectral domains, perhaps the infrared and the optical domains. Let the source flare up for an hour, and only an hour once every century, and let it then be no more luminous than a star of one solar luminosity. And finally, let there be only a thousand of these sources in the Galaxy, only ten of which might be visible at optical wavelengths-the rest being obscured by dust.

The bit rate required to discover these sources is readily calculated. We might wish to comb the sky with pixel sizes one arcsecond on a side. That would mean searching through $\sim 5 \times 10^{11}$ pixels in an all-sky survey. One of these stars might, therefore, be seen to flare up, on average once in ten years, or $\sim 10^{5}$ hours. To be sure that we appropriately characterize the signal, we might wish to make ten measurements on the flare-up in each of the two wavelength bands, during the hour the flare-up lasts, for a total of twenty measurements per hour on the source. In turn, this means that each pixel would need to be observed twenty times per hour, for ten years, before a source of the new kind was discovered. Let twenty bits of information suffice to characterize the range of intensities and positional information for each source, and we see that the total required bit rate is $2 \times 10^{14}$ per hour, or 50 gigabits per second. This is a quite impossible rate, right now.

Of course, it is clear that both the bit rate and the archiving problem can be drastically reduced by proper filtering and data compression. One might choose to make routine measurements that are far coarser, say covering 100 square arc seconds at a time. Only if the total brightness of that area differed appreciably from the expected signal for the patch, based on a complete star map kept in memory, would a more detailed look be taken at this area. This would already reduce the incoming bit rate to a more acceptable gigabit per second level. The rate of transmitting information to a central archive might be further cut by many orders of magnitude, since only differences from the standard maps would need to be transmitted and archived. Still, the example illustrates some of the steps that will be needed in large surveys if rare events are to be discovered in the enormous masses of data that could be expected in principle. Clever compression techniques can also cut the cost of data analysis. 
What I have just described bears relation to the MACHO project. Here one looks for a sudden flaring up of relatively faint sources in arbitrary parts of the sky. One is seeing remote sources lensed by foreground objects which might or might not be as luminous as the lensed source. That project has already registered many terrabytes of information. It is still relatively simple. There may be other phenomena where a source would become apparent only at high spectral resolution, for example if there were short duration bursts of cosmic masers at gamma frequencies. These might not make themselves apparent against the ubiquitous gamma ray background unless searched for with high spectral resolution techniques. To add to the difficulties, if these sources were distant, their systematic red shift would require some form of high resolution multifrequency detection. All this is possible, but it is not likely to become practicable with a single instrument that gathers all these data simultaneously.

\section{Optimization Schemes}

We will need to work out strategies to optimize deep searches. We are not yet sure, even now, whether numerous small but dedicated space missions, can be more effective than observatory class complexes that can cross-correlate a variety of types of information-spatial, spectral, temporal and polarization. And if we do choose the more specialized missions, how should we decide on the most propitious characteristics. Should astrophysical theory determine their make-up, as for example in the SWAS mission to search for and map water vapor, molecular oxygen and a few other chemical species of high astrophysical interest? Or should we build in additional breadth - and at what additional cost - to assure that nothing unexpected has been overlooked? These are important considerations and it is somewhat surprising that little thought has been given to optimization schemes. To date, we have opted almost entirely for either the general observatory class missions, or else for highly dedicated missions, like Hipparcos, radio surveys for $\mathrm{CO}$ or atomic hydrogen, or a search for general relativistic effects such as the Gravity Probe-B.

We do not currently have anything like an optimum search strategy. We may need to consider the best ways of sparse sampling in the temporal, spatial, and spectral domains. These will have to be designed to permit a search for patterns - correlated signals-within and among observations in these domains.

Sparse sampling leaves open the possibility that some rare phenomenon will remain hidden. How can we minimize the risk of that without taking up a far more expensive, exhaustive search? 
Methods of multiplexing, to permit a variety of data to be simultaneously recorded and processed, may provide advantages (c.f. Martin Harwit and Neil J. A. Sloane, 1979). What are the best ways of doing this without prejudging the types of patterns that may emerge?

Just as we in astronomy adopted hand-me-down hardware from the military, throughout the half century since World War II, we may now, in the wake of the Cold War, see what optimization schemes the military and the CIA have developed in their search through enormous data banks they must have accumulated. We have already adopted many of their techniques, but I expect that there is a great deal more that we could take over wholesale without too much expenditure of effort.

Perhaps no optimum strategy exists, and perhaps we are already doing the best we could be expected to. But it might be useful to mount an investigation where such questions could be discussed. It would be different from the ten-year national academy studies, in that there would be no winning mission to emerge. Rather, the investigation would aim to articulate a general philosophy, most probably independent of wavelength range, and therefore not hotly contested by astronomers with different wavelength interest and preferences. It might be a philosophy that would take into account our astrophysical understanding at any given epoch in the development of astronomy, and feed that back to arrive at a progressively more informed way of choosing the next set of optimized steps.

Spending some thought in developing a broad philosophy of this kind, with suggestions to science administrators for sensibly applying it, could turn out to be one of the most effective ways of getting our money's worth from expensive surveys.

\section{Acknowledgements}

The author's work is supported by two grants from NASA.

\section{References}

Harwit, M. "Cosmic Discovery", Basic Books, New York, 1981

Neugebauer, G. and Leighton, R.B. "Two Micron Sky Survey", NASA SP-3047, Washington, D.C., 1969.

ESA Report to COSPAR 1996, page 36

Harwit, M. and Sloane, N.J.A. "Hadamard Transform Optics", Academic Press, 1979 